اثر افزودن يوسته خارجى برنج بر عملكرد، صفات لاشه، فراسنجههاى بيوشيمى

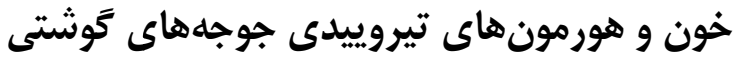

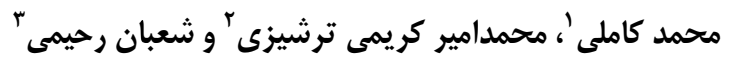

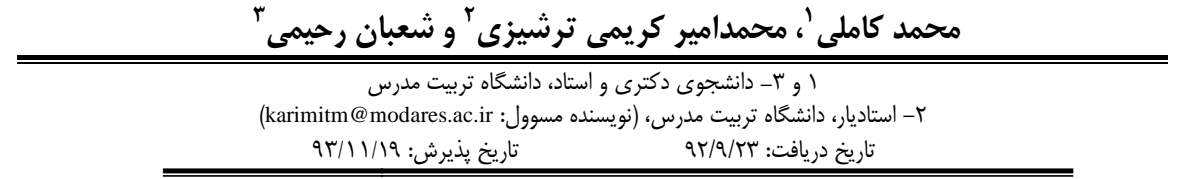

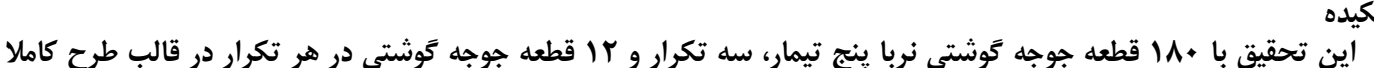

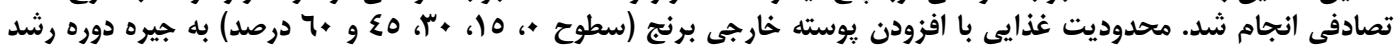

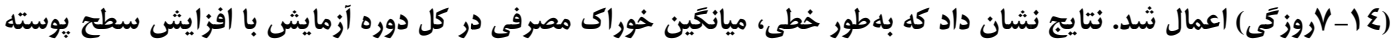

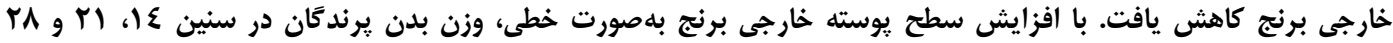

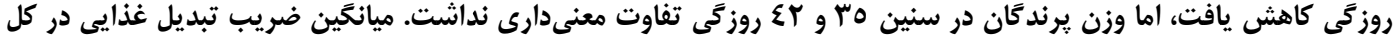

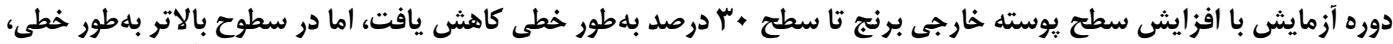

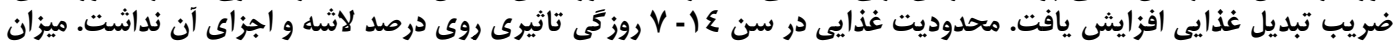

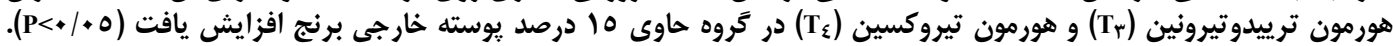

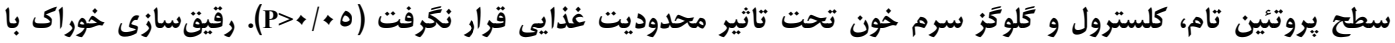

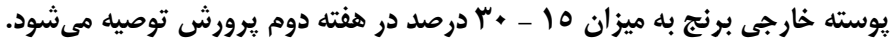
وازههاى كليدى: يوسته خارجى برنج، جوجه توشتى، صفات عملكردى، محدوديت غذايى، هور مونهاى تيروييدى

لاشه نيز كاسته مىشود. اين نظريه امكان استفاده از

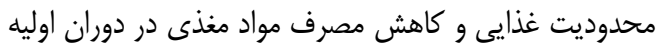

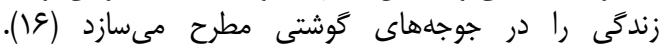

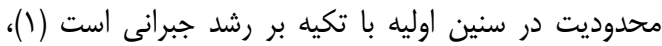

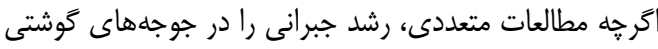

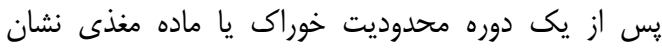

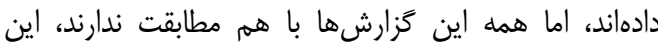

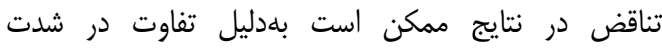

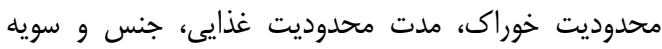

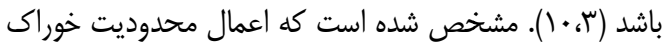

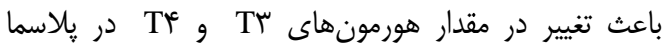

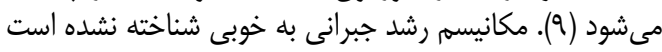

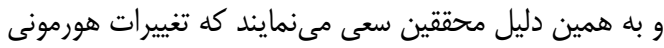

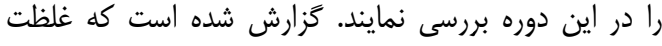

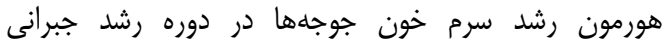

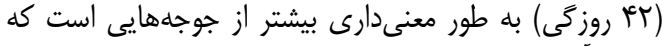

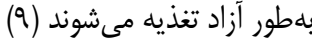

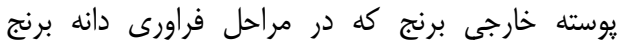

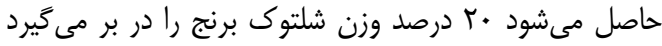

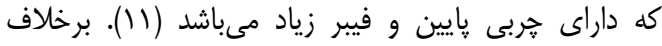

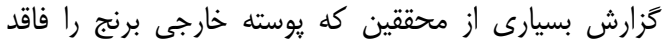

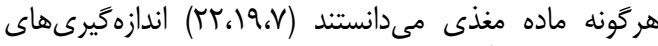

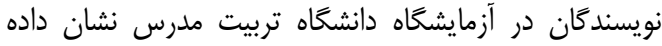

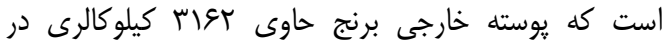

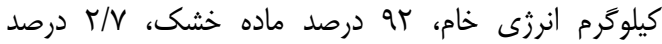

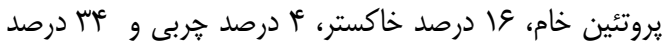

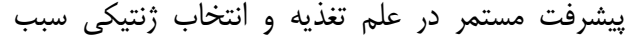

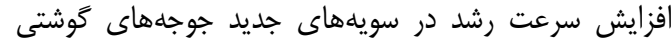

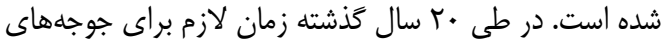

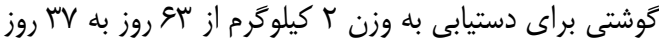

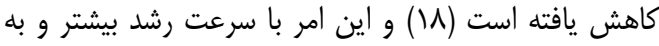

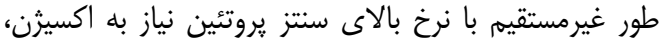

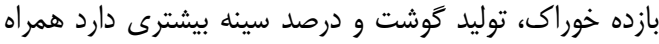

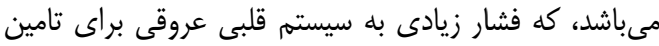

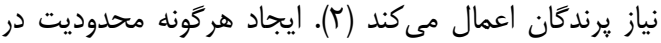

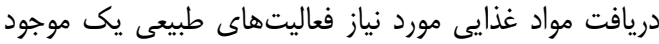

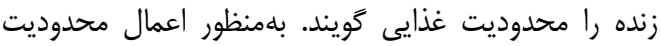

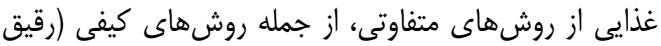

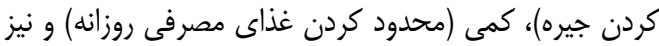

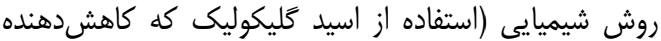

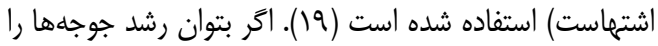

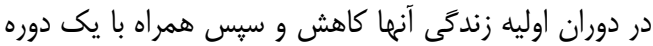

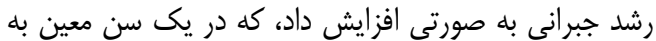

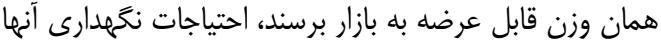

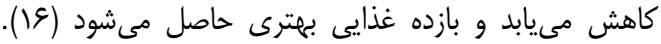

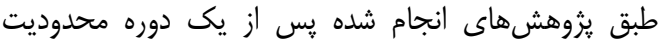

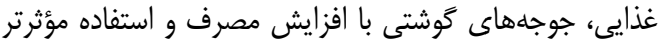

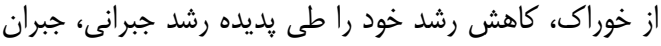

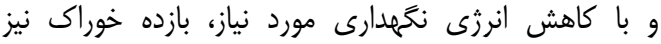

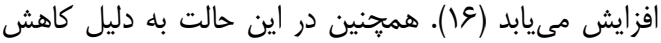

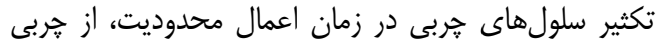




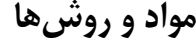

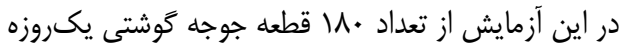

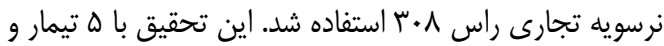

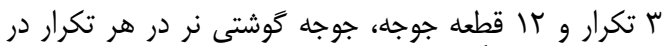

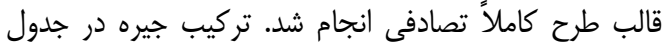

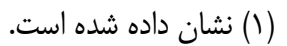

فيبر خام مىباشد. از آنجايى كه سالانه نزديك به سه ميليون

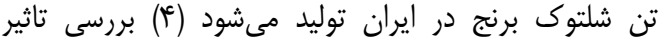

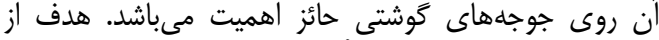

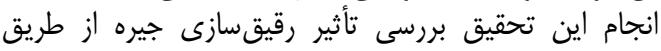

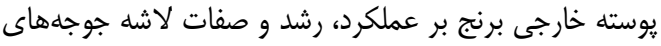
كوشتى بوده است.

جدول ا- تركيب جيرههاى آزمايشى مورد استفاده در تغذيه جوجههاى گَشتى Table 1. Composition of experimental diets fed to broiler chickens

\begin{tabular}{|c|c|c|c|c|c|c|c|c|}
\hline & & \multicolumn{5}{|c|}{ سطوح افزودن يوسته خارجى برنج (درصد) } & & \multirow[b]{2}{*}{ كروههاى آزمايشى } \\
\hline & & 9. & $F \Delta$ & $r \cdot$ & 10 & $\cdot$ & & \\
\hline te-tit & $10-r \Delta$ & & & $1-1 f$ & & & $1-V$ & دوره سنى (روز) \\
\hline 8$) / 1$ & $9.1 \cdot$ & $r m / q f$ & Tr/ar & $51 / \wedge q$ & $\Delta \cdot / \wedge \Delta$ & 9.1. & $\Delta H / S$ & دانه ذرت \\
\hline$T r / \Delta$ & $M F / \Delta$ & IT/AV & $19 / \cdot V$ & $r F / T \Lambda$ & $r q / 4 e^{2}$ & $r F / \Delta$ & $r q / \wedge$ & كنجاله سويا (بَث/\%) \\
\hline . & - & s. & ia & r. & 10 & • & - & يوسته خارجى برنج \\
\hline$r / I V$ & $1 / v 9$ & $\cdot / V \cdot F$ &.$/ 98 \wedge$ & 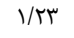 & $1 / 49$ & $1 / v 9$ & $r / M \Lambda$ & روغن سويا \\
\hline $1 / \mathrm{r} \Lambda$ & $1 / 199$ & .1098 &.$/ 119$ & $1 / \cdot{ }^{2}$ & $1 / T \&$ & $1 / 19$ & $1 / V$ & دى كلسيمفسفات \\
\hline 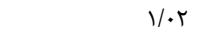 & $1 / \cdot \Delta$ &.$/ 24$ & $\cdot / \Delta V V$ &.$/ V T \Delta$ &.$/ 199$ & $1 / \cdot 0$ & $1 / \mu$ & كربنات كلسيهم \\
\hline 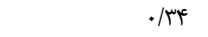 & 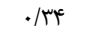 & ع (1//. &.$/ \Lambda A V$ & 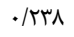 &.$/ 4 \wedge 9$ & $\cdot /$ KF & $\cdot / \mathrm{T} \Delta$ & نمك طعام \\
\hline.$/ T \Delta$ & $\cdot / T \Delta$ & $\cdot / T \Delta$ & $\cdot / T \Delta$ & $\cdot / T \Delta$ &.$/ 4 \Delta$ & $\cdot / T \Delta$ & $\cdot / T \Delta$ & مكمل ويتامينى * \\
\hline$\cdot / T A$ & $\cdot / T A$ &.$/ T \Delta$ & $\cdot / T \Delta$ & $\cdot / T \Delta$ &.$/ T \Delta$ & $\cdot / T A$ & $\cdot / T \Delta$ & مكمل معدنى ** \\
\hline - & $\cdot / 1$ & $.1 \cdot 4$ & $\cdot / \cdot \Delta \Delta$ & $\cdot 1 \cdot \mathrm{V}$ & $\cdot 1 \cdot \wedge \Delta$ &.$/ 1$ & - & ال-ليزين \\
\hline.$/ \mathrm{IV}$ & $\cdot /$ TE & .1 .98 & 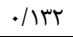 &.$/ 19 \Lambda$ & $\cdot / T \cdot t^{c}$ & $\cdot / T F$ & 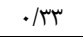 & دىال-متيونين \\
\hline r... & rafi & 1199 & TIDV & rFIN & TEV & rqfi & TMM & انرزى قابل متابوليسم (kcal/kg) \\
\hline $19 / 99$ & $T \cdot / \Delta T$ & $\mid r / \Delta F$ & $\mid f / \Delta V$ & $18 / \Delta 1$ & $\mathrm{~N} / \mathrm{QV}$ & $T \cdot / \Delta T$ & Tr/QT & يروتئين خام (درصد) \\
\hline$\cdot / 4 \wedge$ & $\cdot / \Delta \Delta$ &.$/ 4 t$ & $\cdot / \mu \cdot$ &.$/ 49$ &.$/ F V$ & $\cdot / \Delta \Delta$ & .198 & متيونين (درصد) \\
\hline$\cdot|\wedge|$ & $\cdot / \mathrm{M}$ & 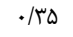 & $\cdot / \uparrow \wedge$ & $\cdot \mid a t$ & $\cdot / V \Delta$ & $\cdot / M$ & $1 / \cdot r$ & متيونين+ سيستين (درصد) \\
\hline 1/. & $1 / 10$ &.$/ 48$ & س سع/. & $\cdot|\mathrm{A}|$ & .191 & $1 / 10$ & $1 / r V$ & ليزين (درصد) \\
\hline$\cdot / \Lambda$ & $\cdot / \Lambda t$ & 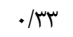 & $\cdot / F \Delta$ & $\cdot / \Delta \Lambda$ & $\cdot / \mathrm{V}$ & $\cdot / A T$ & $\cdot / 199$ & ترئونين (درصد) \\
\hline$\cdot / \mathrm{Vq}$ & $\cdot / \Lambda \mathrm{V}$ & $\cdot / r v$ &.$/ 19 q$ & . & $\cdot / V^{e}$ & $\cdot / \mathrm{AV}$ & $1 / \cdot 0$ & كلسيم (درصد) \\
\hline • & שt/א & $\cdot / 41$ & . T & Tr/ & 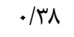 & سז/א. & $\cdot / \Delta$ & فسفرقابل دسترس (درصد) \\
\hline.$/ 10$ &.$/ 1 \mathrm{~V}$ & $\cdot 1 \cdot V$ & .1 .9 &.$/ 14$ &.$/ 10$ &.$/ 1 V$ & $\cdot / 11$ & سديم (درصد) \\
\hline 1.141 & 1.94. & ๑ด१V & squs & ATVD & 99.9 & 1.94. & $1 \cdot \Delta A F^{F}$ & قيمت (ريال در كيلوگرم) \\
\hline
\end{tabular}

اندازهيرى مقدار هورمونهاى تيروييدى و فراسنجههاى

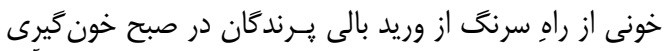

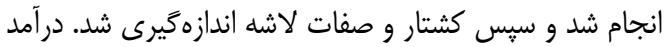
حاصل از فروش گوشت جوجه گوشتى، هزينه

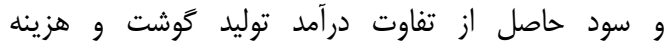
خوراكشاخصهاى اقتصادى بررسى گرديد. با توجه به به سطوح

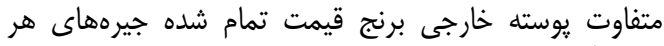

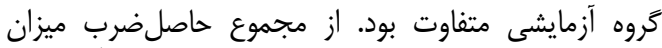

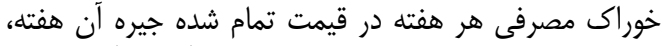

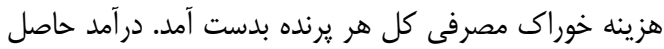

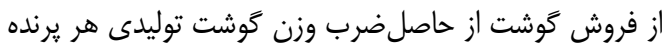

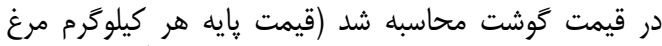

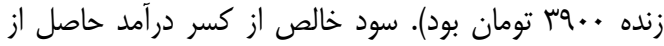

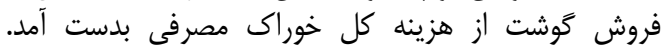

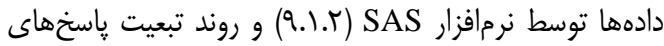

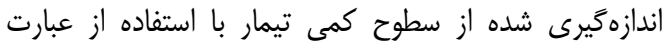
contrast
محدوديت غذايى در سن أI-V روزگى اعمال شد. بعد از

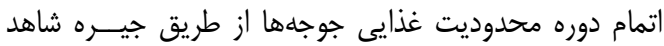

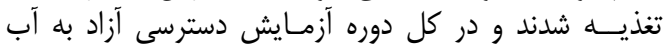

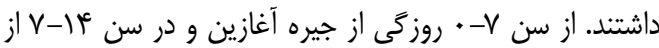

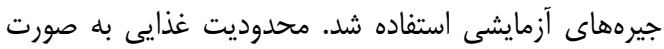

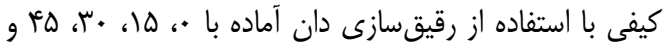

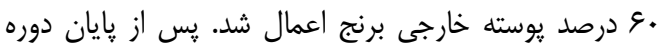

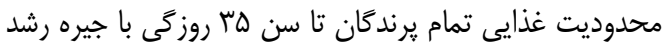

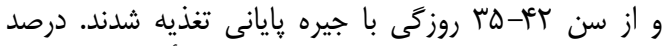

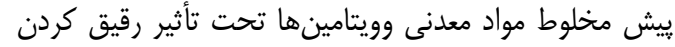

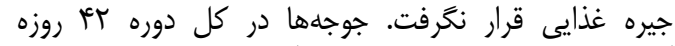

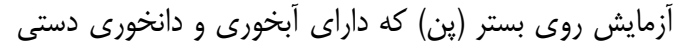

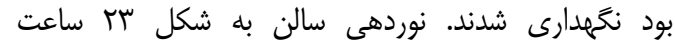

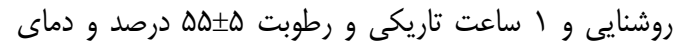

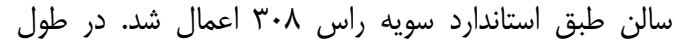

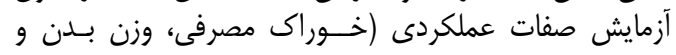

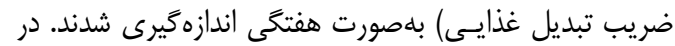

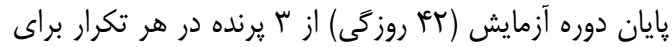


كمتر جوجههاى تحت محدوديت در مقايسه با جوجههاى

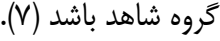

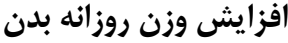

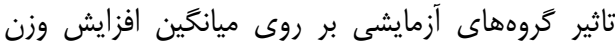

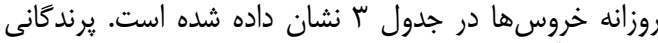

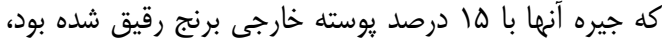

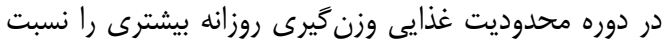

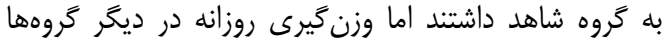

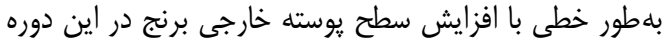

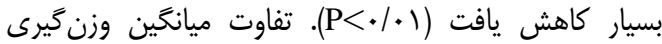

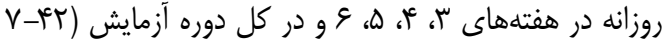

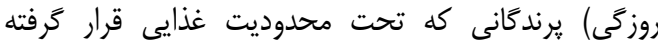

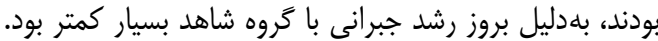

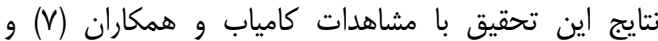
حسن آبادى و همكاران (ه) مطابقت دارد.

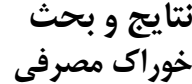

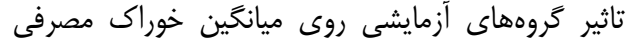

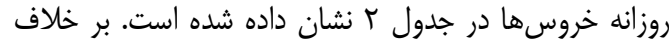

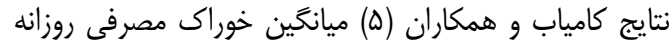

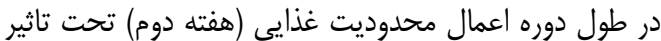

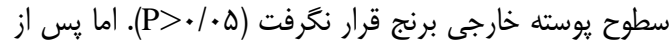

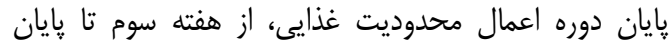

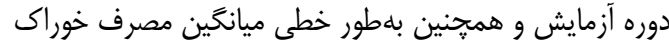

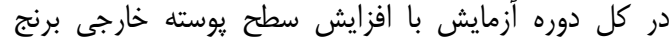

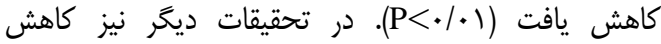

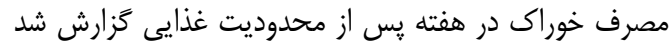

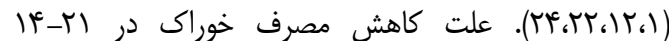

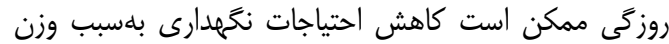

جدول ז- تاثير گَروههاى آزمايشى بر ميانكين خوراى مصرفى روزانه جوجههاى گَوشتى (كرم به ازاى هر يرنده در روز) Table 2. Effect of experimental treatments on average daily feed intake of broiler chickens (g/d)

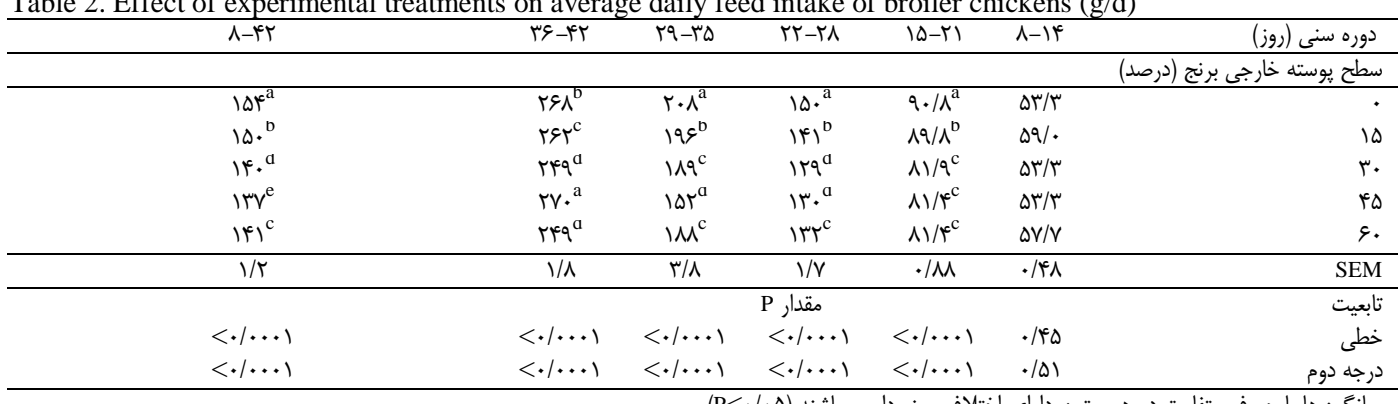

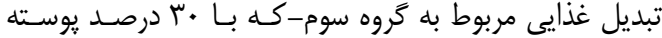

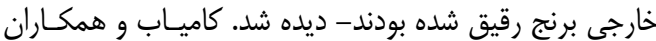

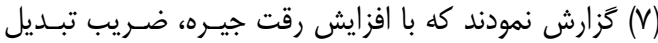

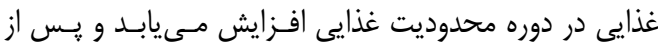

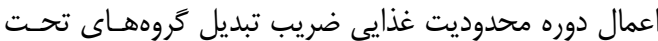

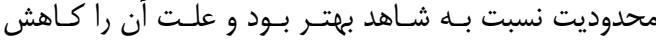

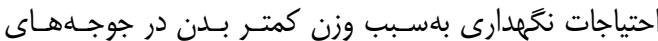

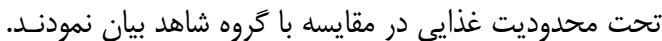

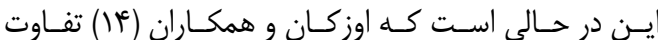

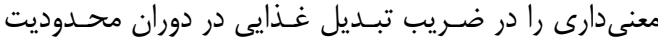

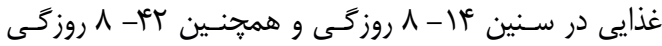
مشاهده نكردند.

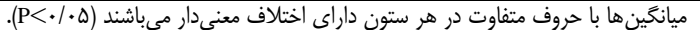

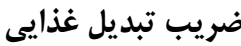

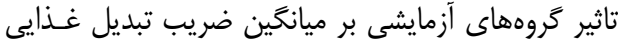

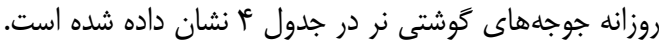

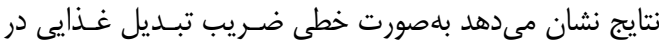

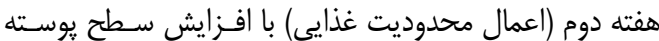

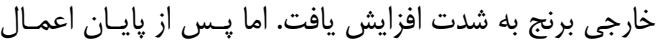

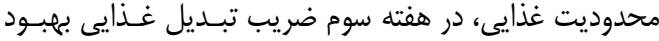

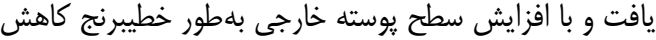

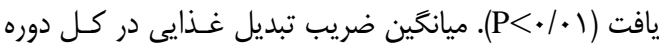

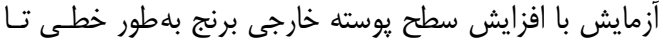

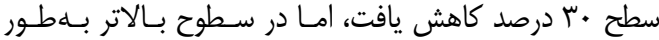

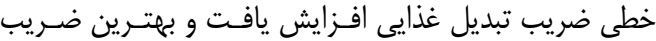

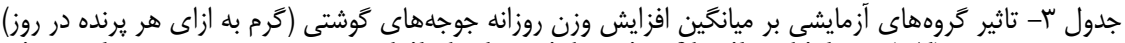
Table 3. Effect of experimental treatments on average daily body weight gain of broiler chickens (g/d)

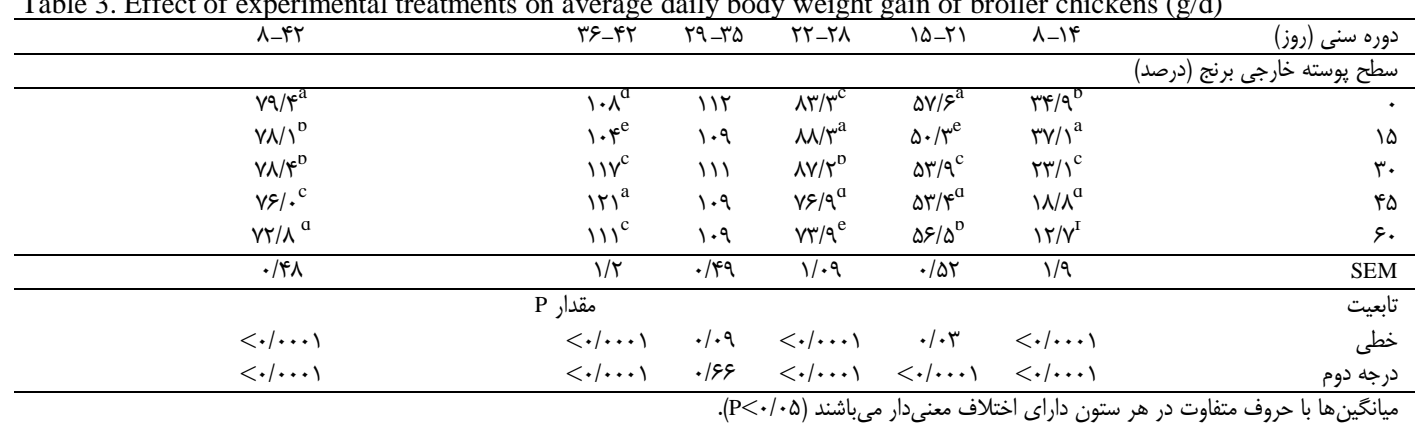


همكاران (ه) نشان دادند كه رقيقسازى خوراك جوجهاهاى

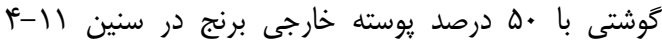

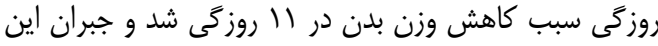

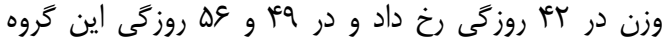

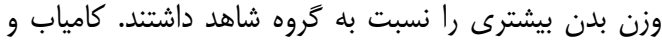

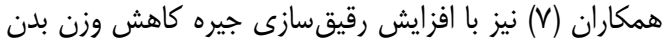

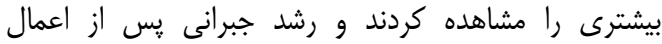

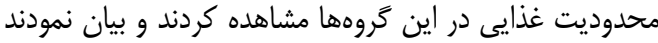

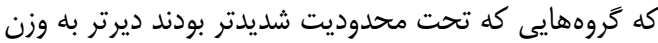

كروه شاهد رسيدند.

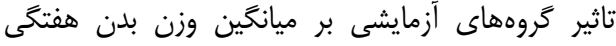

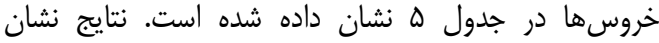

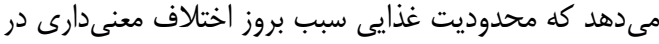

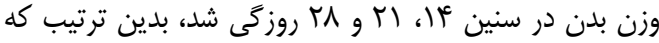

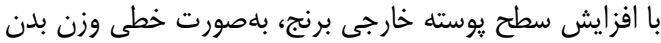

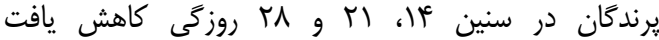

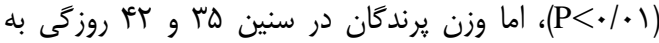

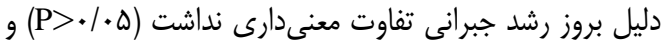

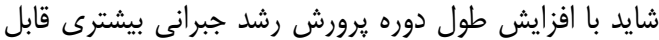
مشاهده بود. حسن آبادى و نصيرى مقدم (و) و حسن آبادى و و برى

جدول عا تاثير گروههاى آزمايشى بر ميانخين ضريب تبديل غذايى روزانه جوجههاى گوشتى Table 4. Effect of experimental treatments on average daily feed conversion ratio of broiler chickens (g/d)

\begin{tabular}{|c|c|c|c|c|c|c|}
\hline A-YY & re-tit & rq-ra & Tr-TA & $|\Delta-Y|$ & $\Lambda-1 f^{2}$ & دوره سنى (روز) \\
\hline & & & & & & سطح يوسته خارجى برنج (درصد) \\
\hline $1 / \Lambda F^{C}$ & $T / 4 \xi^{\circ}$ & $1 / \wedge \Delta^{a}$ & $1 / \lambda \cdot^{a}$ & $1 / V \Delta^{0}$ & $1 / \Delta r^{11}$ & $\cdot$ \\
\hline $1 / \Lambda \Delta^{c}$ & $r / \& q^{a}$ & $1 / \mathrm{v} \Lambda^{0}$ & $1 / 1^{\mathrm{c}}$ & $1 / \mathrm{v} \Lambda^{\mathrm{a}}$ & $1 / \Delta 9^{a}$ & 10 \\
\hline $1 / \wedge r^{\mathrm{a}}$ & $r / M 1^{a}$ & $1 / 99^{c}$ & $1 /\left.\Delta\right|^{a}$ & $1 /\left.\Delta\right|^{c}$ & $r / r \cdot^{c}$ & r. \\
\hline $1 / q \Psi^{D}$ & $r / T Y^{c}$ & $1 / \mathrm{rq}^{\mathrm{a}}$ & $1 / 99^{\circ}$ & $1 / \Delta T^{c}$ & $r / \Lambda \Gamma^{D}$ & is \\
\hline$r / \mu^{a}$ & $r / r \varphi^{c}$ & $1 / \mathrm{N}^{\mathrm{c}}$ & $1 / v \Lambda^{a}$ & $1 / 4 \kappa^{\mathrm{a}}$ & 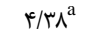 & 9. \\
\hline .1 .4 &.$/ \cdot r$ &.$/ \cdot \mu$ &.$/ . r$ &.$/ . \mathrm{r}$ & $\cdot|T|$ & SEM \\
\hline & & & Pقدار P P & & & تابعيت \\
\hline$<\cdot|\cdots|$, & $<\cdot / \cdots, \mid$ & $\langle\cdot / \cdots|$, & 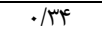 & $\langle\cdot / \cdots+|$ & $\langle\cdot / \cdots+|$ & خطى \\
\hline$\langle\cdot|\cdots|$ & $\langle\cdot| \cdots$, & $\langle\cdot|\cdots|$, & $\langle\cdot|\cdots|$, & $\langle\cdot /, \ldots|$ & $\langle\cdot|+\cdots|$ & درجه دوم \\
\hline
\end{tabular}

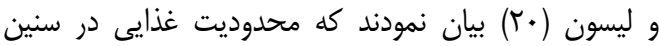

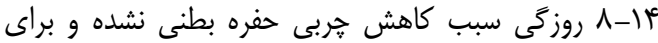

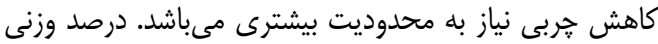

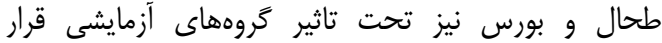

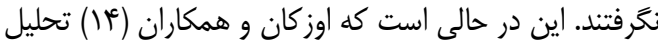

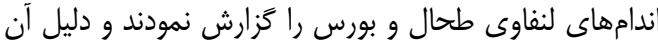

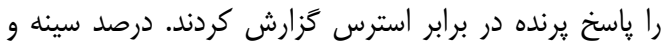

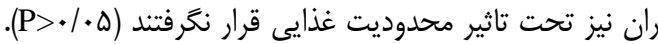

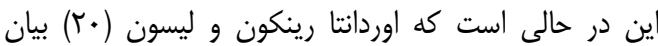

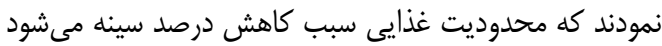

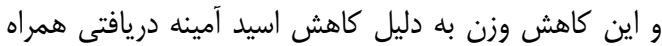

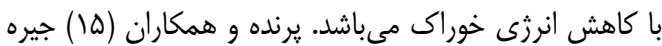

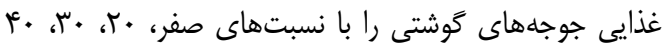

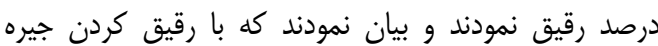

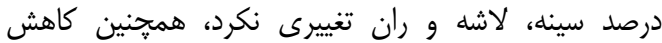

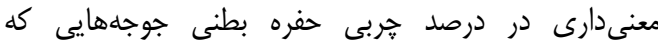

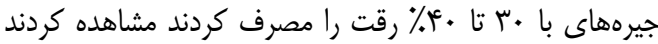

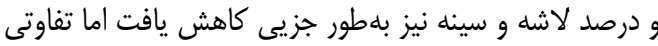
در درصد ران مشاهده نكردند.

هورمونهاى تيروئيدى دئي

تاثير گروههاى آزمايشى بر هوريدى هورمونهاى تيروئيدى خون

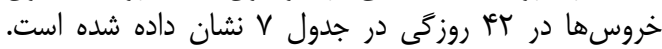

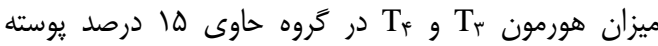

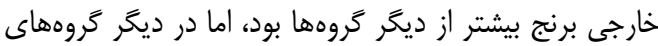

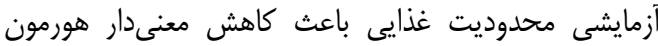

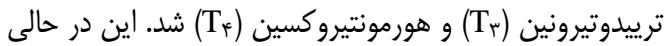

زوبير و ليسون (مها بيان كردند كه افزايش مطلق مصرف

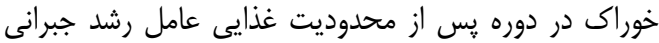

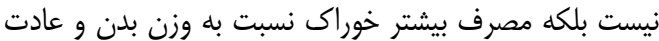

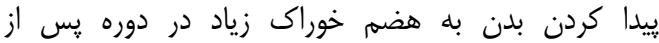

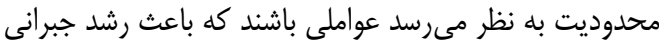

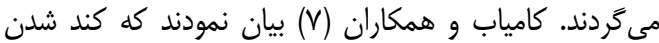

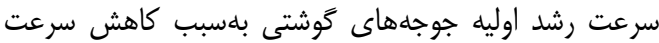

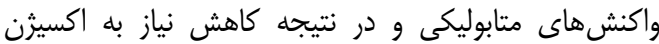

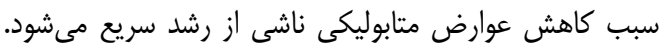

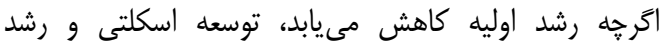

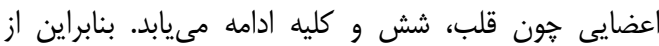
لحاظ فيزيولوزيكى طيور توانايى بيشترى براى تحلى تحمل استرس

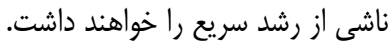

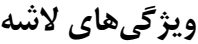

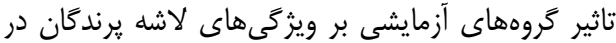

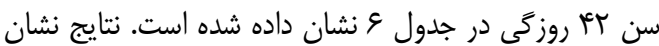

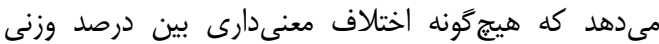

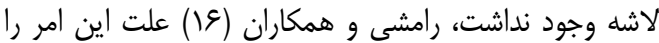

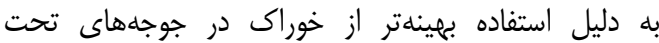

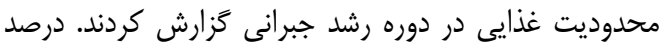

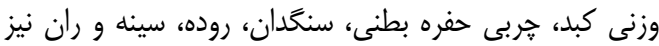

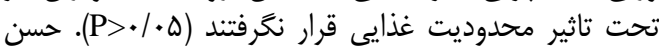

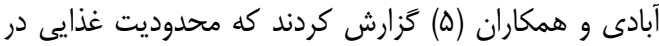

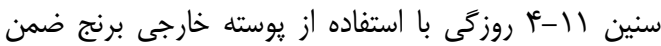

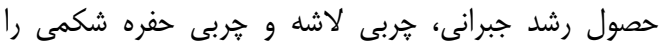
كاهش مى دهد. اما رضايى و همكار جئ لان (19) و اوردانتا رينكون حفره 


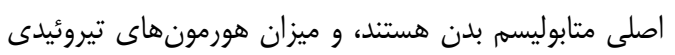

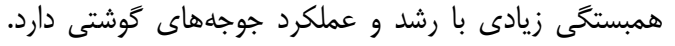

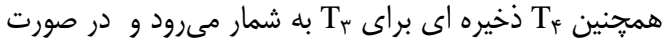

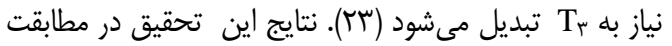

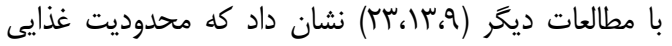

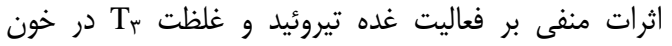
داشت و سبب كاهش غلظت ائ فواليت غوه تيرويل ومون شد.
است كه مك كورتى و همكاران (Iا) بيان نمودند كه

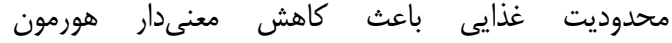

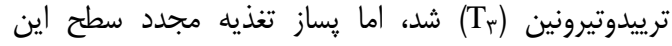

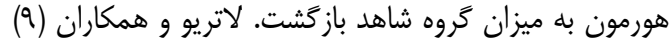

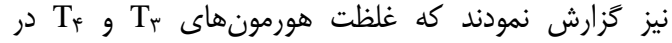

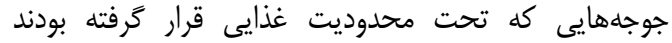

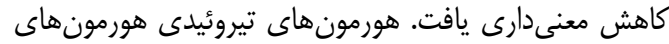

جدول ه- تاثير گروههاى آزمايشى بر ميانخين وزن بدن جوجههاى گوشتى (گرم)

Table 5. Effect of experimental treatments on average body weight of broiler chickens (g)

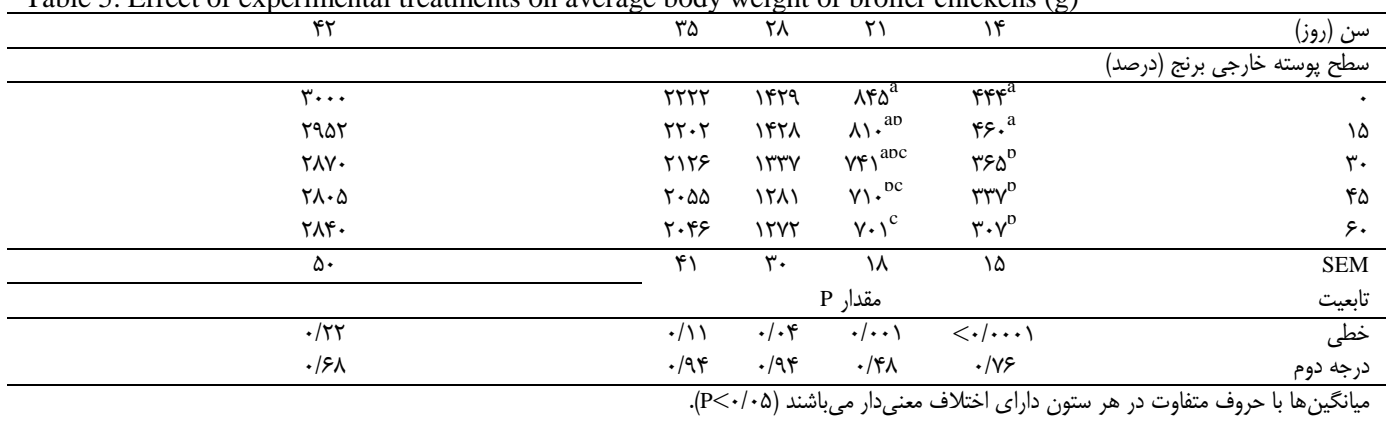

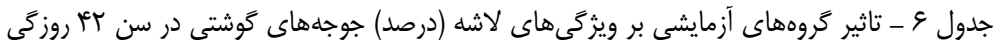
Table 6. Effect of experimental treatments on carcass characteristics of broiler chickens on 42 d (\%)

\begin{tabular}{|c|c|c|c|c|c|c|c|c|c|}
\hline ران & سينه & روده & سنكَدان & هربى حفره بطنى & بورس فابرسيوس & طحال & كبد & لاشه & كروههاى آزمايشى \\
\hline$r . / 19$ & $r \Delta / \Delta T$ & $\Delta / F)$ & $1 / \Delta 1$ & $1 / 11$ & $\cdot / T^{f}$ &.$/ 1$ & $1 / \wedge \Delta$ & $V I / \% \Delta$ & . \\
\hline$r .1 . r$ & $r \varepsilon / \Lambda$. & $\Delta / \cdot q$ & $1 / \Delta V$ &.$/ 98$ & $\cdot|r|$ & $\cdot / f^{f}$ & שr/T & VT/RT & 10 \\
\hline$r \cdot \pi r$ & $r \Delta / \Delta \varphi$ & $\Delta / Q Y$ & $1 / 4 F^{2}$ & $1 / r$. &.$/ N V$ &.$/ \pi r$ & $r / \cdot \Delta$ & $V T / \Delta S$ & r. \\
\hline $19 / 19$ & rg/Aq & $r / g T^{\circ}$ & $1 / r \Lambda$ & $1 / \Delta F$ &.$/ 1 V$ & .1 .9 & $r / \cdot r$ & $r / I r$ & is \\
\hline $19 / 49$ & re/.r & $r / A V$ & $1 / \Delta F$ & $1 / 1 F$ &.$/ N V$ & سו/. & $r / T$. & $15 / 19$ & 9. \\
\hline$\cdot / r$ & $\cdot / \Psi V$ & $\cdot / N \mu$ & $\cdot / \cdot f^{r}$ & $\cdot 1 \cdot \mathrm{V}$ & .1 .1 & $\bullet \cdot \cdot V$ & .1 .9 & . T & SEM \\
\hline \multicolumn{9}{|c|}{ مقدار P } & تابعيت \\
\hline$\cdot / / r$ &.$/ 99$ &.$/ .0$ & . & $\cdot / \mu \mid$ & .1 .9 & $\cdot / \varepsilon \phi$ & $\cdot / \pi f$ & $\cdot|q|$ & خطى \\
\hline$\cdot / \Lambda \Delta$ & .18. & $\cdot / T^{F}$ &.$/ \% q$ &.$/ 8$ &.$/ \% q$ &.$/ 95$ & .199 &.$/ 1 r$ & درجه دوم \\
\hline
\end{tabular}

جدول V - تاثير گروههاى آزمايشى بر هورمونهاى تيروييدى و فراسنجهاى خونى جوجه گوشتى نر در بأ روزگى Table 7. Effect of experimental treatments on thyroid hormones and blood factors of broiler chickens

\begin{tabular}{|c|c|c|c|c|c|c|}
\hline 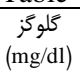 & 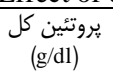 & $\begin{array}{l}\text { كلسترول } \\
\text { (mg/dl) }\end{array}$ & $\begin{array}{c}\text { ترى كليسريد } \\
\text { (mg/dl) }\end{array}$ & $\mathrm{T}_{4}(\mathrm{ng} / \mathrm{ml})$ & $\mathrm{T}_{3}(\mathrm{ng} / \mathrm{ml})$ & سطح يوسته خارجى برنج (درصد) \\
\hline $1 V^{\mu}$ & $F / \Delta T$ & $1 \Lambda$. & $1 F T^{\circ}$ & $4 \cdot 119^{\circ}$ & $r / \varphi^{c}$ & . \\
\hline r.r & سع/א & $r \cdot e^{2}$ & $\mid Q \Lambda^{\mathrm{a}}$ & a./ $/ \mathrm{r}^{\mathrm{a}}$ & $\varphi / r \Lambda^{\mathrm{a}}$ & 10 \\
\hline 194 & $f / 99$ & 119 & $100^{a}$ & $r \cdot / k r^{a}$ & $r / 9)^{e}$ & r. \\
\hline IVA & $r / \Delta T$ & 199 & $1 f q^{a}$ & e & $r / \cdot q^{\mathrm{a}}$ & is \\
\hline IAV & $F / r y$ & 197 & $1 Q T^{a}$ & $F \cdot / V^{c}$ & $r / 99^{\circ}$ & 9. \\
\hline$\Gamma / \uparrow \wedge$ & .1 .4 & $r / q \Lambda$ & $1 / \pi$ & $\cdot / \Delta$ & .1 .9 & SEM \\
\hline \multicolumn{6}{|c|}{ Pقدار P P } & تابعيت \\
\hline$\cdot / \mathrm{AV}$ &.$/ T 4$ & $\cdot|\Delta|$ &.$/ . r \mathrm{r}$ & $<\cdot|\cdots|$ & $<\cdot / \cdots+\mid$ & خطى ( خ خ \\
\hline .1 .9 & .1 .9 & سب/. & .1 .91 & $<\cdot|\ldots|$ & $<. / \ldots . \mid$ & درجه دوم \\
\hline
\end{tabular}




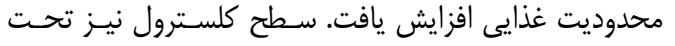

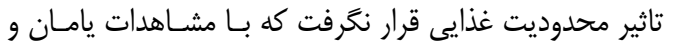

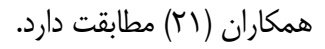

مقايسه سود اقتصادى

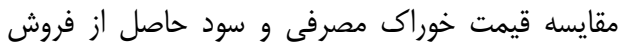

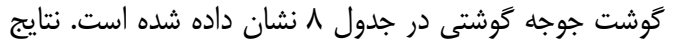

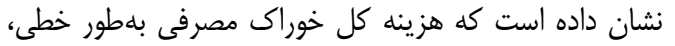

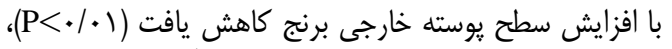

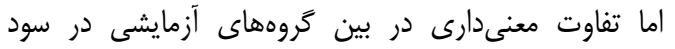

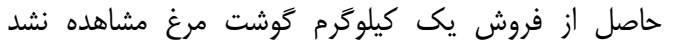

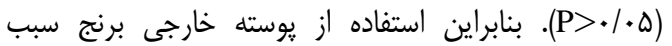

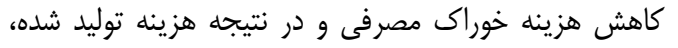

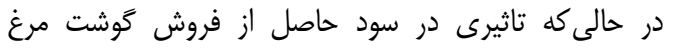

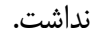

فراسنجههاى خونى

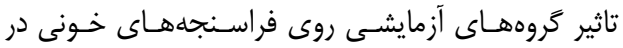

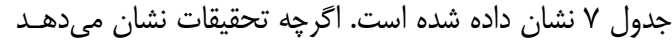

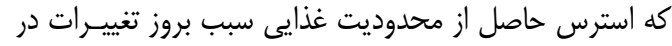

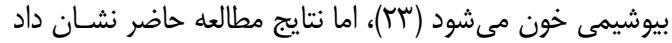

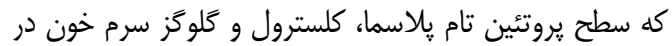

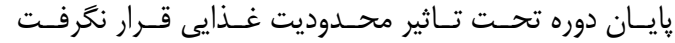

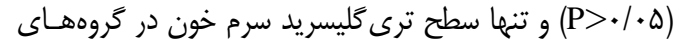

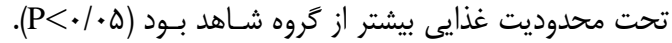

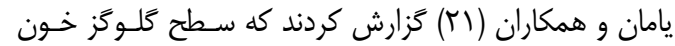

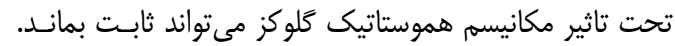

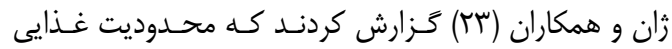

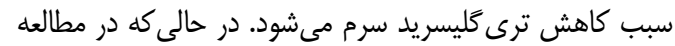

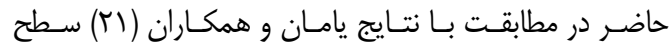

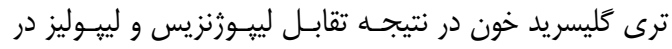

جدول ^- مقايسه هزينه خوراك مصرفى و سود حاصل از فروش گوشت مرغ زنده (ريال) Table 8. Effect of experimental treatments on feeding cost and profit of selling live birds (Rilas)

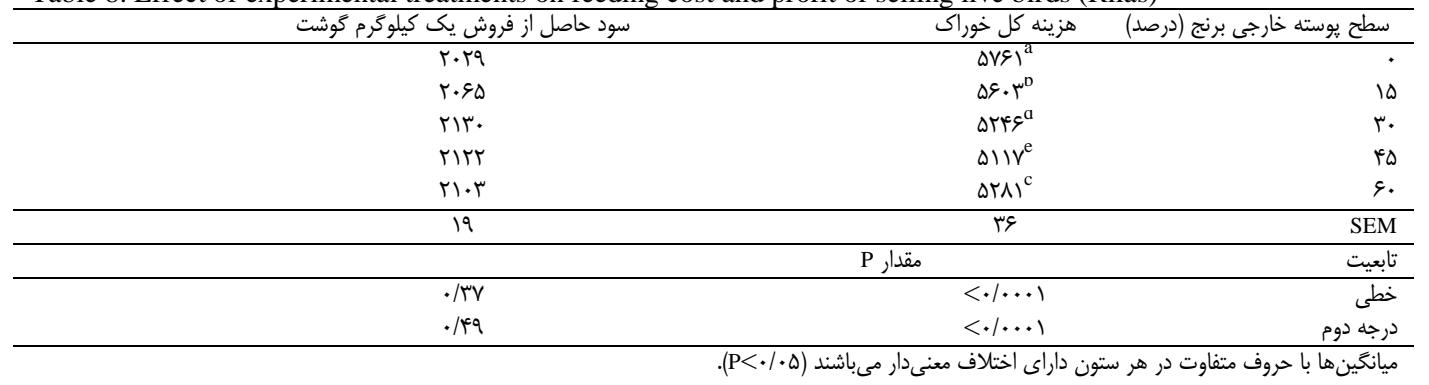

نداشت، حاصل شد. طبق نتايج به دست آمده رقيقسازى

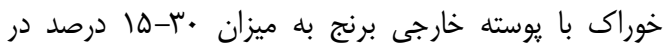
هفته دوم يرورش توصيه مى شود.
بطور كلى نتايج اين آزمايش نشان ميدهد كه مهائ محدوديت

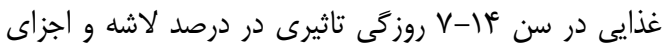

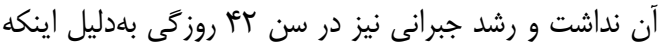

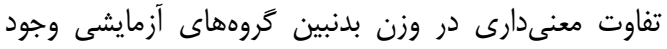


1. Acar, N. F.G. Sizemore, G. R. Leach, R.F. Wideman, R.L. Owen and G.F. Barbato. 1995. Growth of broiler chickens in response of feed restriction regiments to reduce as cites. Poultry Science, 74: 833843.

2. Baghbanzadeh, A. and E. Decuypere. 2008. Ascites syndrome in broilers: Physiological and nutritional perspectives. Avian Pathology, 37: 117-126.

3. Balog, J.M., B.D. Kidd, W.E. Huff, G.R. Huff, N.C. Rath and N.B. Anthony. 2003. Effect of cold stress on broilers selected for resistance or susceptibility to ascites syndrome. Poultry Science, 82: 1383-1387.

4. Food and Health. 2011. World rice Trading. Retrieved December 15, 2012, from http://www.worldfood.ir/ T_22623.

5. Hassanabadi, A., A. Golian and H. Nassiri Moghaddam. 2009. The effect of early feed restriction on performance and serum thyroxin concentration of broiler chickens. Iranian Journal of Animal Science Research, 1: 57-66 (In Persian).

6. Hassanabadi, A. and H. Nassiri Moghaddam. 2006. Effect of early feed restriction on performance characteristics and serum thyroxin of broiler chickens. International Journal of Poultry Science, 5: 1156-1159.

7. Kamyab, A.R., K. Yussefi and M. Rezaei. 2003. Performance of broiler chickens during and following feed restriction at an early age. Iranian Journal of Agriculture Science, 34: 19-28 (In Persian).

8. Kaps, M. and W.R. Lamberson. 2004. Biostatistics for Animal Science CABI Publishing, $459 \mathrm{pp}$.

9. Lauterio, T.J. and C.G. Scanes. 1987. Hormonal responses to protein restriction in two strains of chickens with different growth characteristics. Journal of Nutrition, 117: 758-763.

10. Lee, K.H. and S. Leeson. 2001. Performance of broilers fed limited quantities of feed or nutrients during seven to fourteen days of age. Poultry Science, 80: 446-454.

11. Leeson, S. and J.D. Summer. 2008. Commercial Poultry Nutrition. 3rd edn., Nottingham University Press, Nottingham, UK, 398 pp.

12. Leeson, S., J.D. Summers and L.J. Caston. 1991. Diet dilution and compensatory growth in broilers. Poultry Science, 70: 867-873.

13. McMurtry, J.P., I. Plavnik, R.W. Rosebrough, N.C. Steele and J.A. Proudman. 1988. Effect of early feed restriction in male broiler chicks on plasma metabolic hormones during feed restriction and accelerated growth. Comparative Biochemistry and Physiology, 91: 67-70.

14. O"zkan, S., I. Plavnik and S. Yahav. 2006. Effects of early feed restriction on performance and ascites development in broiler chickens subsequently raised at low ambient temperature. Journal of Applied Poultry Research, 15: 9-19.

15. Parande, R., A. Nikkhah and A.M. Yosef Hakimi. 2000. Effect of energy and protein dilution in starter and grower period on performance, carcass characteristics and compensatory growth in broilers. Pajouhesh and Sazandegi, 13: 116-119 (In Persian).

16. Rameshi, F., M. Eslami and J. Fayazi. 2007. Effect of wet rations on performance of broiler chicken after feed restriction in compensatory growth. Pajouhesh and Sazandegi, 74: 46-52 (In Persian).

17. Rezaei, M., A. Teimouri, J. Pourreza, H. Sayyahzadeh and P.W. Waldroup. 2006. Effect of diet dilution in the starter period on performance and carcass characteristics of broiler chicks. Journal of Central European Agriculture, 7: 63-69.

18. Sahraei, M. and F. Shariatmadari. 2007. Effect of different levels of diet dilution during finisher period on broiler chickens performance and carcass characteristics. International Journal of Poultry Science, 6: 280-282.

19. Toghiani, M., A. Samie and A. Gheisari. 2003. Effect of early feed restriction and increased nutrient density after restriction period on compensatory growth in broiler chickens. Journal of Science and Technology of Agriculture and Natural Resources, 7: 151-159 (In Persian).

20. Urdaneta-Rincon, M. and S. Leeson. 2002. Quantitative and qualitative feed restriction on growth characteristics of male broiler chickens. Poultry Science, 81: 679-688.

21. Yaman, M.A., K. Kita and J. Okumura. 2000. Different responses of protein synthesis to refeeding in various muscles of fasted chicks. British Poultry Science, 41: 224-228.

22. Yussefi Kelaricolaii, K., A. Kamyab and M. Rezaei. 2003. Performance of broiler chickens during and following feed restriction at early age. Journal of Science and Technology of Agriculture and Natural Resources, 6: 117-126 (In Persian).

23. Zhan, X.A., M. Wang, H. Ren, R.Q. Zhao, J.X. Li and Z.L. Tan. 2007. Effect of early feed restriction on metabolic programming and compensatory growth in broiler chickens. Poultry Science, 86: 654660.

24. Zubair, A.K. and S. Leeson. 1994. Effect of varying period of early nutrition restriction on growth compensation and carcass characteristics of male broilers. Poultry Science, 73: 129-136.

25. Zubair, A.K. and S. Leeson. 1996. Compensatory growth in the broiler chicken: A review. World's Poultry Science, 52: 189-201. 


\title{
Effect of Supplementing Rice Hull on Performance, Carcass Characteristics, Blood Biochemistry and Thyroid Hormones of Male Broiler Chickens
}

\author{
Mohammad Kamely ${ }^{1}$, Mohammad Amir Karimi Torshizi ${ }^{2}$ and Shaban Rahimi ${ }^{3}$ \\ 1 and 3- Ph.D. Student and Professor, Tarbiat Modares University \\ 2- Assistant Professor, Tarbiat Modares University \\ (Corresponding author: karimitm@modares.ac.ir) \\ Accepted: February 8, 2015 \\ Received: December 14, 2013
}

\begin{abstract}
This study was performed by five treatments and three replicates using 180 Ross male broilers (12 birds in each pen) in a completely randomized design. Feed restriction was applied by supplementing the rice hull levels $(0,15,30,45$ and 60 percent). To grower feed ( 7 to 14 days of age). Average feed intake had a linear decrease with increasing in rice hull levels. Body weight was decreased linearly in 14, 21 and 28 days of age, but due to compensatory growth there were not any significant differences in body weight on 35 and 42 days. Average of feed conversion ratio decreased by increasing in rice hull levels up to 30 percent but in higher levels feed conversion ratio increased linearly. Feed restriction during 7-14 days of age has any significant differences in carcass percentage and its parts. Concentration of plasma triiodothryronine $\left(\mathrm{T}_{3}\right)$ and thyroxine $\left(\mathrm{T}_{4}\right)$ increased in $15 \%$ rice hull groups $(\mathrm{P}<0.05)$. Blood total protein, cholesterol and glucose were not affected by feed restriction $(\mathrm{P}>0.05)$. Feed dilution by rice hull up to 15-30 percentages in the second week of broiler production is recommended.
\end{abstract}

Keywords: Broilers, Feed Restriction, Performance, Rice Hull, Thyroid's hormones 